\title{
Pathochistological and immunohistochemical study in small intestine metastasis as first clinical expression of invasive lobular breast carcinoma: Prognosis and complex treatment
}

\author{
Lena Marinova ${ }^{*}$; Bistra Yordanova²; Nikolay Evgeniev \\ ${ }^{1}$ Department of Radiotherapy, Complex Oncology Center, Ruse, Bulgaria. \\ ${ }^{2}$ Clinical Pathology Department, Complex Oncology Center, Ruse, Bulgaria. \\ ${ }^{3}$ Department of Medical Oncology, Complex Oncology Center, Ruse, Bulgaria.
}

*Corresponding Author: Lena Marinova

Department of Radiotherapy, Complex Oncology

Center, Ruse, Bulgaria.

Email: rad_marinova@abv.bg

Received: Apr 05, 2021

Accepted: Apr 30, 2021

Published: May 05, 2021

Archived: www.jjgastro.com

Copyright: (C) Marinova L (2021).

\section{Abstract}

The gastrointestinal metastases (GIM) of breast carcinoma (BC) are a rare clinical finding, even more rarely diagnosed as initial symptoms at unknown BC. We present a 50-year-old woman with metastases in the terminal ileum combined with multiple bone metastases from an unknown invasive carcinoma of left mammary gland. Gastrointestinal metastases are rarely observed in invasive lobular breast carcinoma. Differential diagnosis between intestinal metastases from primary BC and primary intestinal neoplasm is extremely difficult. Determination of tumor pathohistogenesis require careful pathohistological and immunohistochemical analysis. Complex treatment follows the primary neoplasm principles. The prognosis in such clinical cases is unfavorable, but after timely complex oncological treatment, including emergency operation, chemotherapy, hormone and target therapy, 36 monthly survival is achieved.

Keywords: gastrointestinal metastases; invasive lobular breast carcinoma; pathohistological analysis; immunohistochemical analysis; complex treatment. 
Citation: Marinova L, Yordanov B, Evgeniev N. Pathochistological and immunohistochemical study in small intestine metastasis as first clinical expression of invasive lobular breast carcinoma: Prognosis and complex treatment. Japanese J Gastroenterol Res.

$2021 ; 1(1): 1001$

\section{Introduction}

Invasive breast carcinoma $(\mathrm{BC})$ is the most commonly diagnosed neoplasm in women representing a serious threat to life [1]. Over the past two decades, due to improved early diagnosis and complex treatment, mortality from BC has decreased [2-4]. Prolonged survival increases distant metastases [1]. Hematogenic metastases from the $\mathrm{BC}$ in the gastrointestinal tract are relatively rarely diagnosed. The most common covered organs are colon and rectum, followed by the stomach and small intestine [5]. Breast cancer is one of the most common malignancies that metastasize to the gastrointestinal tract (GIT), along with melanoma, ovarian and bladder cancer [6]. Invasive lobular breast carcinoma (ILBC) is the second at frequency histological type, diagnosed in $12 \%$ $-20 \%$ of the invasive BC [6-8]. Distant hematogenic metastases of the ILBC are diagnosed too rarely in the GIT, the peritoneum, the retroperitoneum, the uterine body, the ovaries and the skin [7, 9-11].

\section{Clinical case}

Clinical case is about a 50-year-old woman, who has been admitted in surgical department with complaints of difuse abdominal pain, stronger in the upper floor, nausea without vomiting and defecation of scarce rare faeces. Partial resection of the small intestine with ileostomia and lymphatic biopsy of increased lymph nodes in the bowel radius has been performed on 21.12.2018. After histological examination and immunohistochemical analysis, it was found to be a distant metastases from a invasive lobular breast carcinoma (ILBC). The latter finding was verified with a core needle biopsy of the left mammary gland.

Local status before the abdominal surgery: Abdomen above the chest level, ballooned, palpatory soreness in the upper floor with moderately expressed muscle resistance and data on tympanism.
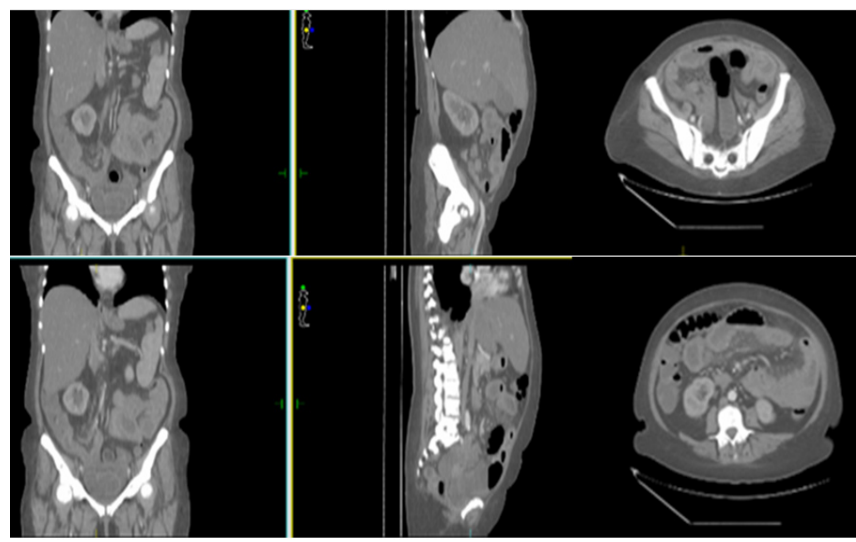

Figure 1: Preoperative abdomenal CT: Circumferential thickening of the small intestinal walls, mainly jejunum, and saved areas in the small intestine. The expanded bowel loops of mixed type formed hydroaeric levels.
Abdominal echography: Liver with normal echogenic structure and smooth outlines, without visible lesions and suspected data on a pathological cocarda in colon descendens.

Preoperative abdomenal CT with venous contrast: There are expanded bowel loops of mixed type, with the predominance of the slurry with formed hydroaeric levels (Figure 1). There is a circumferential thickening of the small intestinal walls, mainly jejunum, and saved areas in the small bowel. Lytic-sclerotic bone lesions of thoracic and lumbar vertebrae and pelvic bones, most likely bone metastasis.

Intraoperative exploring: There is a plenty of serous blood collapse in the abdomen. The secretion in the small pelvis is with a gelatinous structure. In the terminal ileum of about $20 \mathrm{~cm}$ from the ileocecal valve, there are three tumor formations in the intestinal wall of different sizes from 2 to $3 \mathrm{~cm}$, and the proximal formation caused a full stop of the passage. It distantly observing expanded intestinal loops with a heavily thickened wall and whitish points of serose nodules. In the intestinal radix, increased lymph nodes of different diameter to $2-3 \mathrm{~cm}$ are reported. Liver and spleen are increased by retail grain surface. There was decompression of the proximal part of the small intestine from the stop with a subsequent partial resection of a portion of the terminal ileum of about $40 \mathrm{~cm}$. Both ends appeared as an ilesome two-point on the right of the abdominal wall. Biopsy of part of the small bowel and lymph nodes from intestinal radix was taken.

Macroscopic description: Small intestine specimen, a full of about $30 \mathrm{~cm}$ with four whitish seals in different areas of it with a diameter of up to $2 \mathrm{~cm}$. Covering the entire thickness of the intestinal wall, stanning almost completely lumen. The mucous membrane in the sealing area is visibly intact.

Histological result: Microscopic description- In the described areas, infiltration of monomorphic cells, in places forming trabecules engaging mucosa, muscle layer, subset tissue and one of the infiltration areas in the adjacent fat. After revision of the histological result - a small intestinal wall with preserved forced architectonics, foci mucosa and infiltration in lamina propria, submucosa, muscularis propria and serosa from dischostered monomorphic cells with trabecular growth, minimal nuclear polymorphism and dispersed chromatin, tumor emboli in lymphatic vessels (Figure 2).

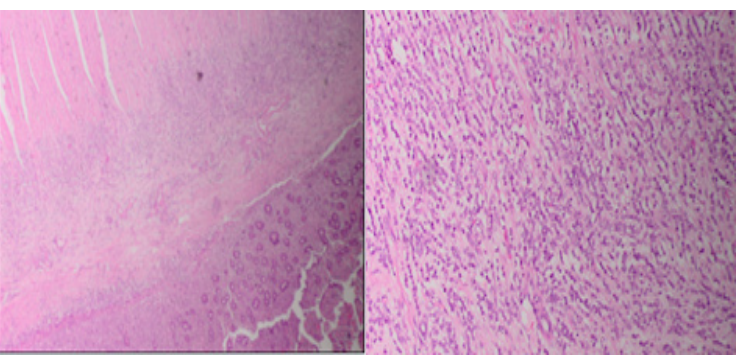

Figure 2: Photomicrography of metastasis in small intestine from invasive lobular carcinoma / H\&E x20, H\&E x100. 
Immunohistochemical (IHC) analysis: The tumor population indicates a negative reaction for CD 56, CD45, Chromogranin A, E-Cadherin, CD 3, CD20 and focal Sinaptophysine positiveness (Figure 3). Average level of proliferative tumor cell index (Ki67$15 \%)$ is reported. Diffuse strong IHC expression for Cytokerin, AE1 / AE3 clone, diffuse moderately positive reaction for estrogen receptor (ER) in tumor cell nuclei, negative IHC expression for progesterone receptor (PR) and HER2 status, as well as focal positive expression for Mammaglobin in the cytoplasm of tumor cells is reported (Figure 4). In the mesenteric lymph nodes of the intestinal radix, metastases from invasive lobular carcinoma were detected (Figure 5). From IHC of lymphatic metastases proved a highly positive reaction for Cytokerin, AE1 / AE3 and Mammaglobin, and moderately positive expression for ER (Figure 6, Figure 7).

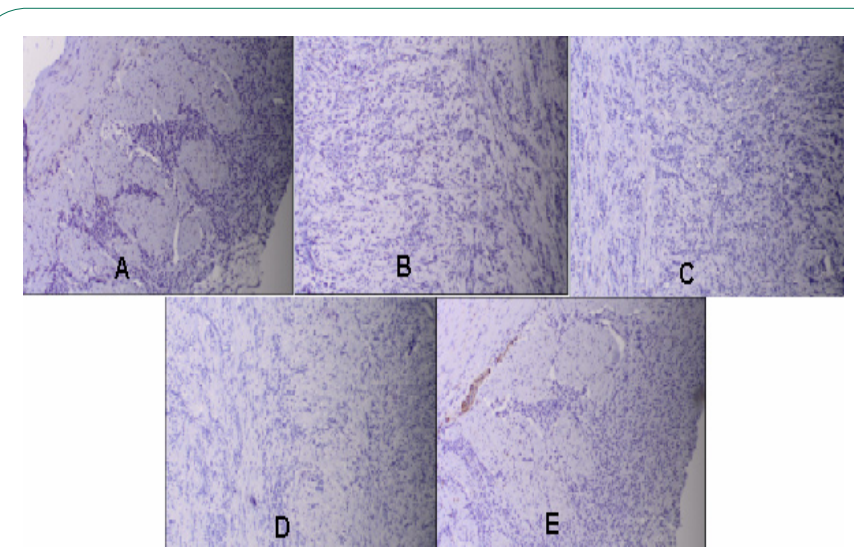

Figure 3: Photomicrography of immunohistochemistry - The tumor population indicates negative expression for $A /$ Chromogranin $A$; B/ E-cadherin; C / CD 3; D / CD20; E / Focal positive expression for Synaptophysine in the axons of peripheral nerves and cells with neuroendocrine differentiation in the mucose of the small intestine $\times 20$

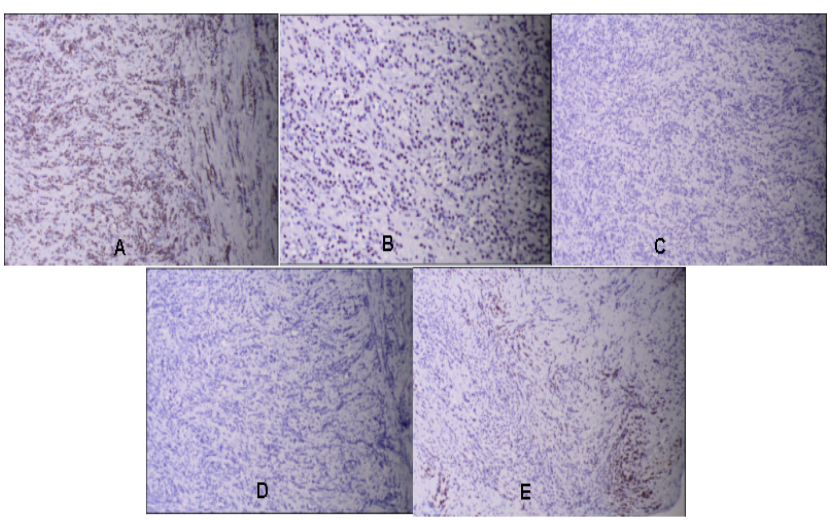

Figure 4: Photomicrography of immunohistochemistry - A / Diffuse strong expression for Cytokeratin, AE1 / AE3 branch; B / Diffuse moderate positive reaction in the tumor cell nuclei for ER; C / Negative reaction for PR; D / negative reaction for HER2 status; E / Focal positive expression in tumor cell cytoplasm for Mammaglobin x20.

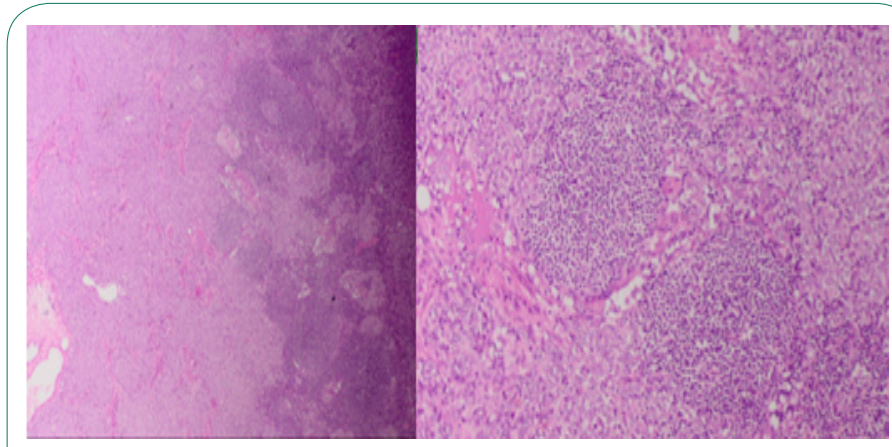

Figure 5: Photomicrography of metastases in a mesenteric lymph node / H\&E x20, H\&E x100.

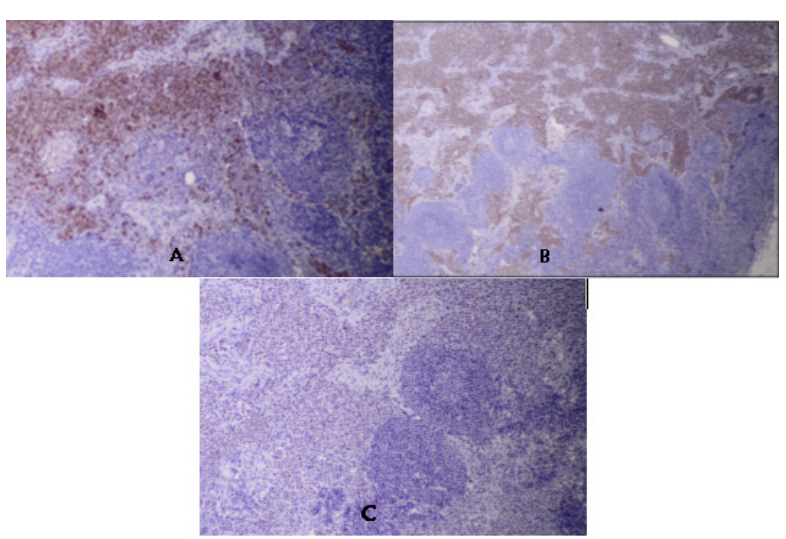

Figure 6: Photomicrography of immunohistochemical analysis of a mesenteric lymph node A / highly positive reaction for Cytokerin, AE1 / AE3; B / positive reaction for Mammaglobin; C/ moderately positive expression for ER $\times 20$.

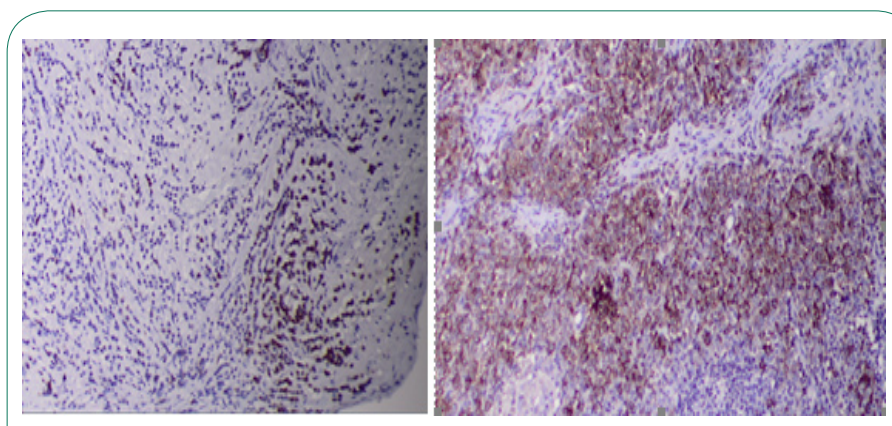

Figure 7: Photomicrography of immunohistochemical expression for Mamaglobin: A / Focal positive expression in the wall of the small intestine; $\mathrm{B} /$ Diffuse positive expression in the metastatic mesenteric lymph node $x 100$.

From the core biopsy from the left breast tumor, invasive lobular carcinoma with the following immunohistochemical characteristics: moderate positive expression for ER $(4+2)=6$ and negative reaction for PR and HER2 was verified (Figure 8 ). 


\section{A}

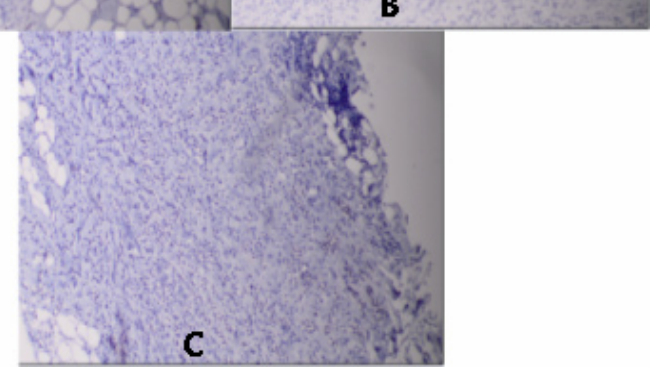

Figure 8: Photomicrography of immunohistochemical analysis of the left breast biopsy - A / Moderate positive expression for ER; C / Negative reaction for PR; C / Negative reaction for HER2.

The basis of morphology and IHC study can be assumed to be suitable for haematogenic metastasis in small intestine and metastases in mesenteric lymph nodes of primary invasive lobular breast carcinoma.

The patient was presented to an Oncological committee and judged for chemotherapy and endocrine therapy. Figure 9 represents the CT image of the left breast carcinoma after the biopsy, and in figure 10 are seen generalized bone metastases on vertebra and pelvic bones.

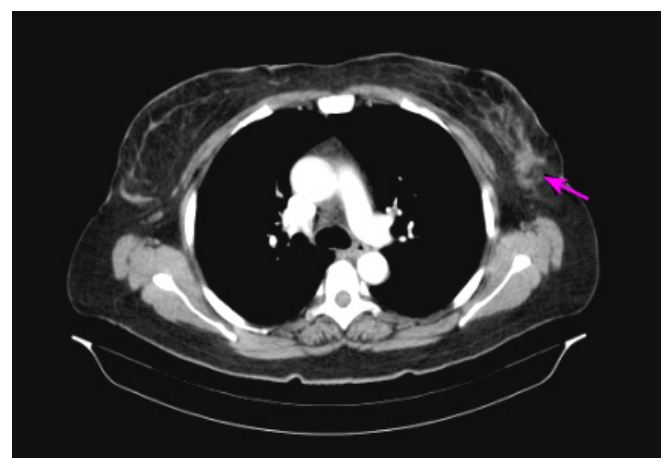

Figure 9: CT image of carcinoma in the left breast.

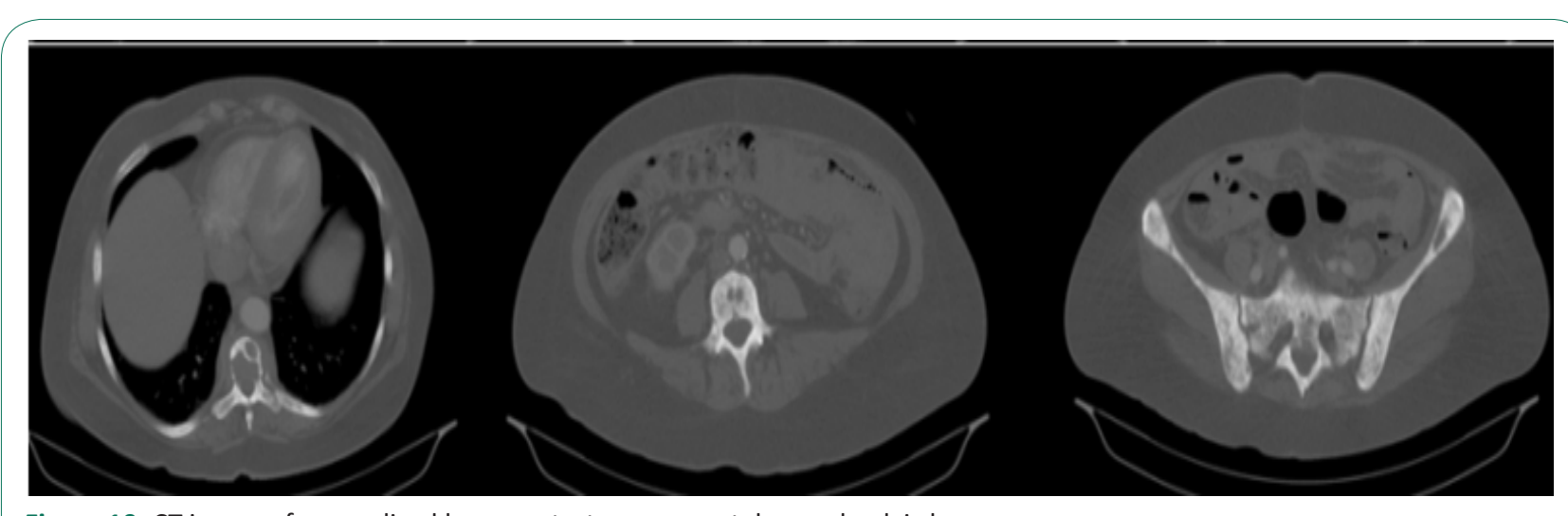

Figure 10: CT image of generalized bone metastases on vertebra and pelvic bones.
Postoperatively 3 courses first line chemotherapy (Ch) were conducted followed with a contrast- enhanced CT scan which showed disease progression with multiple liver metastases (Figure 11). The complex treatment continued with a first-line hormone therapy with Fulvestrant and Denosumab. The patient died after 6 months due to pancytopenia as a consequence of bone marrow infiltration.

\section{Discussion}

Distant haematogenic metastases in breast carcinoma (BC) are diagnosed in $75 \%$ of patients, regardless of the primary treatment or its absence. Rare gastrointestinal metastases (GIM) from BC usually cover the upper gastrointestinal tract as a stomach, small bowel and pancreatic-biliary area [12]. Lobular carcinoma tends to spread to bones, gynecological organs, peritoneum and retroperitoneum, and is less likely to involve the GIT [9-11]. The diagnosis of metastatic lesions to the GIT becomes even more unlikely on the rare occasion when it represents as first manifestation of BC [13]. In the literature, two situations have been described with the expression of unusual metastases from primary BC. The first, which is more commonly observed, is known treated in the past $\mathrm{BC}$ after a prolonged latent period of $16-30$ years $[1,14,15]$. Metastases have been published in the digestive tract in men with proven BC $[16,17]$. Nonspecific symptoms and the prolonged latent period result in the wrong diagnosis of primary intestinal neoplasm [1]. It is much less than the second situation, namely the appearance of GIM as a first clinical manifestation of primary BC [6,18-24]. Intestinal hematogenic metastases may be single or multiple [1]. In progressive expansion of GIM, diffuse organizing and rigids causes stenosis and obstruction [25-27]. Until the diagnosis, primary BC is reached after an acute abdominal syndrome as intestinal obstruction [20,22,28-30], such as the situation in our clinical case.

Interesting is the observed difference in the localization of distant metastases in the two different histological types of BCinvasive ductal carcinoma (IDC) and invasive lobular carcinoma (ILC). IDC has a trend for metastases in liver, lung and brain, and ILC - bones, gynecological organs, peritoneum, retroperitoneum and GIT $[7,31,32]$. ILC, which is only $12 \%$ of primary invasive BC, contributes to $64 \%$ of GIM [5].

The exact mechanisms for the different localization of distant haematogenic metastases from ILC and IDC are not sufficiently clarified [12]. This difference is explained by various studies, much 


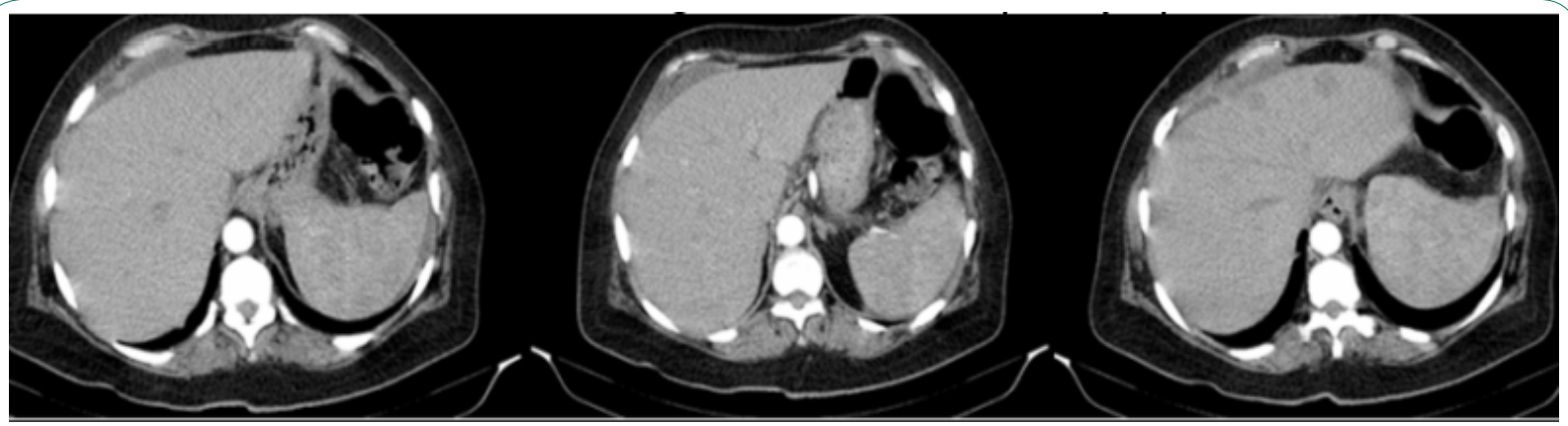

Figure 11: CT image- Disease progression with multiple liver metastases.

of which leans that the cause is due to the small size and form of tumor cells of ILC and the lack of expression for e-Cadherin (factor supporting their adhesion). These cellular factors favor the migration of metastatic carcinoma cells to anatomical structures more favorable to their braking $[7,33,34]$.

For the diagnosis of intestinal metastasis from the primary $\mathrm{BC}$ of great importance is an IHC analysis taking into account the expression of hormonal receptors (Estrogen and Progesterone), HER2 status and Mammaglobin (MGB) (Figures 4,6,7). The Gross Cystic Disease Fluid Protein 15 (GCDFP-15) and the ER and/or PR are positive in metastatic lesions of the breast, in contrast to most colorectal or gastric carcinomas, which are usually negative [6]. The expression of MGB, whose sensitivity in metastatic $B C$ was $55.4 \%$ to $84.3 \%$, higher than that of GCDFP-15 was investigated [23]. In contrast of a number of primary colorectal carcinomas, tumor cells are positive of ER, GCDFP-15 [35], CA153, and cytokeratin (CK) 7 and negative to CK20 and progesterone receptor (PR). This IHC maintains the diagnosis of intestinal metastasis from the BC $[1,6]$. In a newly opened or famous $B C, I H C$ is required to intestinal metastasis with the material from the core biopsy or breast surgery. In our case, full compliance between hormonal status and HER2 status (Figures 4,8) is reported.

For the differential diagnosis of the presented clinical case, there is a distinction of intestinal metastases from other primary tumors of the gastrointestinal tract. After $\mathrm{IHC}$, the tumor cells indicates a negative reaction for CD 56, CD 45, Chromogranin A, E-Cadherin, CD 3, CD 20 and focal reaction for Sinaptophysine (Figure 3). By the immunohistochemical analysis, neuroendocrine tumor and lymphoma of the small intestine were ruled out.

\section{Complex treatment}

Although survival of patients with BC and GIM is increasing, prognosis remains poor. GIM is often associated with extensively disseminated disease [36]. The emergency surgery in the presented clinical case has been found a bowel obstruction caused from three metastases in the wall of the small intestine, along with mesenteric lymph metastasis. Our case indicates that intestinal metastases should be included in the differential diagnostic plan in patients with intestinal symptom and presence of generalized bone metastases, even without a history of breast cancer. Improving survival was observed in selected patients with metastases after palliative surgical interventions (44 months vs. 9 months) [5]. A negative survival effect has advanced age and stomach metastases, and Tamoxifen treatment in positive hormonal receptors improves the prognosis, that is generally unfavorable $[5,22]$. Remissions are reported in $32-53 \%$ of patients [37]. Survival after diagnosis of GIM from primary BC is unfavorable in an average overall survival to one year [22]. Few patients experience 2 years, but the longest survival is 9 years $[38,39]$. In our clinical case, after complex treatment, including emergency operation, chemotherapy (Ch), hormone therapy and targeted therapy, 36 months overall survival is reached. Systemic treatment with $\mathrm{Ch}$, hormone therapy or a combination of both, should be started at any BC metastatic in the GIT [37].

\section{Conclusion}

Hematogenic metastases from the $\mathrm{BC}$ in the gastrointestinal tract are relatively rarely diagnosed. They usually cover the upper GIT as a stomach, small intestine and pancreatic-biliary tract. In the literature, two situations have been described with the expression of unusual GIMs from primary BC. The first, more common, is known treated in the past $\mathrm{BC}$, after a prolonged latent period of 16-30 years. Much lesser is the appearance of GIMs as the first manifestation from primary $\mathrm{BC}$. For the diagnosis of intestinal metastasis from unexplained $\mathrm{BC}$, the immunohistochemical analysis of tumor cells concerning the expression of hormonal receptors (ER and PR), HER2 and Mammaglobin is extremely important. Our case shows that the GIMs should be included in the differential diagnostic plan in patients with intestinal symptoms, even without a history of primary BC. Hematogenic metastases in the GIT of primary BC are related to unfavorable prognosis. This fact requires a timely complex oncological treatment. In the presented patient after emergency operation, chemotherapy, endocrine and targeted therapy, 36 months overall survival is achieved.

\section{References}

1. Min Liu, Lei Zhang, Liang Guo, et al. Intestinal metastasis from breast invasive ductal carcinoma after a long latency: case report and literature review. Onco Targets Ther. 2018; 11: 8599-8603.

2. Early Breast Cancer Trialists' Collaborative Group (EBCTCG) Effects of chemotherapy and hormonal therapy for early breast cancer on recurrence and 15-year survival: an overview of the randomised trials. Lancet. 2005; 365(9472): 1687-1717.

3. Siegel RL, Miller KD, Jemal A. Cancer statistics. CA Cancer J Clin. 2015; 65: 5-29.

4. Berry DA, Cronin KA, Plevritis SK, et al. Effect of screening and adjuvant therapy on mortality from breast cancer. $\mathrm{N}$ Engl J Med.2005; 353(17): 1784-1792.

5. Mclemore EC, Pockaj BA, Reynolds C, et al. Breast cancer: presentation and intervention in women with gastrointestinal metastasis and carcinomatosis. Ann Surg Oncol. 2005; 12(11): 
$886-894$

6. Rodrigo Arrangoiz, Pavlos Papavasiliou, Holly Dushkin, and Jeffrey M. Farma. Case report and literature review: Metastatic lobular carcinoma of the breast an unusual presentation. Int J Surg Case Rep. 2011; 2(8): 301-305.

7. Sastre-Garau X, Jouve M, Asselain B, et al. Infiltrating lobular carcinoma of the breast: clinicopathological analysis of 975 cases with reference to data on conservative therapy and metastatic patterns. Cancer. 1996; 77: 113-120.

8. Rakha EA, El-Sayed ME, Menon S, et al. Histologic grading is an independent prognostic factor in invasive lobular carcinoma of the breast. Breast Cancer Res Treat. 2008; 111: 121-127.

9. Santini D., Altomare A., Vincenzi B., et al. An increase of CA 19.9 as the first clinical sign of ileocecal valve metastasis from breast cancer. In Vivo. 2006; 20:165-168.

10. Aurello P, D'Angelo F, Cosenza G, et al. Gastric metastasis 14 years after mastectomy for breast lobular carcinoma: a case report and literature review. Am J Surg. 2006; 72: 456-460.

11. Ayantunde AA, Agrawal A, Parsons SL, Welch NT. Esophagogastric cancers secondary to a breast primary tumor do not require resection. World J Surg. 2007; 31: 1597-1601.

12. Idrees Khan, Rehan Malik, Amina Khan, et al. Breast Cancer Metastases to the Gastrointestinal Tract Presenting with Anemia and Intra-abdominal Bleed. Cureus. 2017; 9(7): e1429.

13. Van Halteren HK, Peters H, Gerlag PGG. Large bowel mucosal metastases from breast cancer. J Clin Oncol. 1998; 16: 3711-13.

14. Schwarz RE, Klimstra DS, Turnbull AD. Metastatic breast cancer masquerading as gastrointestinal primary. Am J Gastroenterol. 1998; 93(1): 111-114.

15. López Deogracias M, Flores Jaime L, Arias-Camisón I, et al. Rectal metastasis from lobular breast carcinoma 15 years after primary diagnosis. Clin Transl Oncol. 2010;12(2):150-153.

16. Ferrari AB, Pulcini G, Gheza F, et al. Duodenal metastasis from male breast cancer: a case report and review of the literature. J Med Case Rep. 2009; 3: 8331.

17. Koleilat I, Syal A, Hena M. Metastatic male ductal breast cancer mimicking obstructing primary colon cancer. Int J Biomed Sci. 2010; 6(1): 66-70.

18. Arrangoiz R, Papavasiliou P, Dushkin H, Farma JM. Case report and literature review: metastatic lobular carcinoma of the breast an unusual presentation. Int J Surg Case Rep. 2011; 2(8): 301-305.

19. Mouawad NJ, Cleary RK. Small bowel obstruction as the primary presentation of undiagnosed metastatic lobular breast carcinoma. Breast Dis. 2011; 33(1): 35-40.

20. Sato T, Muto I, Hasegawa M, et al. Breast signet-ring cell lobular carcinoma presenting with duodenal obstruction and acute pancreatitis. Asian J Surg. 2007; 30(3): 220-223.

21. Tsujimura K, Teruya T, Kiyuna M, et al. Colonic metastasis from breast carcinoma: A case report. World J Surg Oncol. 2017; 15(1): 124.

22. Savanis G, Simatos G, Tzaida O, et al. Gastrointestinal tract metastasis as first presentation of breast cancer. J BUON. 2006; 11(1): 79-81.

23. Joohyun Woo, Joo-Ho Lee, Kyoung Eun Lee, Sun Hee Sung,
Woosung Lim. Gastric Metastasis as the First Presentation One Year Before Diagnosis of Primary Breast Cancer. Am J Case Rep. 2018; 19: 354-359.

24. Eljabu W, Finch G, Nottingham J, Vaingankar N. Metastatic deposits of breast lobular carcinoma to small bowel and rectum. Int J Breast Cancer. 2011:4139-49.

25. Okido $\mathrm{M}$, Seo $\mathrm{M}$, Hamada $\mathrm{Y}$, et al. Metastatic breast carcinoma simulating linitis plastica of the colon: Report of a case. Surg Today. 2011; 41(4): 542-545.

26. Law WL, Chu KW. Scirrhous colonic metastasis from ductal carcinoma of the breast: Report of a case. Dis Colon Rectum. 2003; 46(10): 1424-1427.

27. Voravud N, El-Naggar AK, Balch CM, Theriault RL. Metastatic lobular breast carcinoma simulating primary colon cancer. Am J Clin Oncol. 1992; 15(4): 365-369.

28. Calò PG, Fanni D, lonta MT, Medas F, Faa G, Atzori F. Jejunal obstruction caused by metastasis from an undiagnosed breast cancer: A case report. Tumori. 2012; 98(3): 89-91.

29. Carcoforo P, Raiji MT, Langan RC, et al. Infiltrating lobular carcinoma of the breast presenting as gastrointestinal obstruction: A mini review. J Cancer. 2012; 3: 328-332.

30. Kandil E, King S, Alabbas H, Moroz K, Wright M. Image of the month. Small-bowel metastasis from infiltrating lobular breast cancer. Arch Surg. 2009; 144(10): 973-974.

31. Harris $M$, Howell $A$, Chrissohou $M$, et al. A comparison of the metastatic pattern of infiltrating lobular carcinoma and infiltrating ductal carcinoma of the breast. Br J Cancer. 1984; 50: 23-30.

32. Lamovec J, Bracko M. Metastatic pattern of infiltrating lobular carcinoma of the breast: an autopsy study. J Surg Oncol. 1991; 48: 28-33.

33. Lehr HA, Folpe A, Yaziji H, Kommoss F, Gown AM. Cytokeratin 8 immunostaining pattern and E-cadherin expression distinguish lobular from ductal breast carcinoma. Am J Clin Pathol. 2000; 114(2): 190-6.

34. Critchley AC, Harvey J, Carr M, Iwuchukwu O. Synchronous gastric and colonic metastases of invasive lobular breast carcinoma: case report and review of the literature. Ann R Coll Surg Engl. 2011; 93(5): e49-e50.

35. Schwarz RE, Klimstra DS, Turnbull AD. Metastatic breast cancer masquerading as gastrointestinal primary. Am J Gastroenterol. 1998; 93(1): 111-4.

36. Hannes Ruymbeke, Luc Harlet, Barbara Stragier, et al. Anorectal metastasis from breast carcinoma: A case report and review of the literature. BMC Res Notes. 2018; 11: 268.

37. Deogracias M, Jaime L, Camisón I, Zamacola I, Murillo Guibert J, Suescun García R. Rectal metastasis from lobular breast carcinoma 15 years after primary diagnosis. Clin Transl Oncol. 2010; 12: 150153.

38. Taal BG, den Hartog Jager FCA, Steinmetz R, Peterse H. The spectrum of gastrointestinal metastases of breast carcinoma. Gastrointest Endosc. 1992; 38: 136-141.

39. Bamias A, Baltayiannis G, Kamina S. Rectal metastases from lobular carcinoma of the breast: Report of a case and literature review. Ann Oncol. 2001; 12: 715-718. 